\title{
Hifema traumático: seguimento de um ano
}

\author{
Traumatichyphema:one-year follow-up
}

\author{
Karolinne Maia Rocha ${ }^{1}$ \\ Dinorah Piacentini Eng'el ${ }^{1}$ \\ Filipe B. Accioly de Gusmão \\ Elisabeth Nogueira Martins ${ }^{2}$ \\ Nilva Simeren Bueno de Moraes ${ }^{3}$
}

\section{RESUMO}

Objetivos: Analisar retrospectivamente a evolução de pacientes portadores de hifema decorrente de trauma ocular contuso quanto à acuidade visual inicial e final, aumento da pressão intra-ocular, ocorrência de ressangramento, tempo de absorção do coágulo, e necessidade de cirurgia. Métodos: Foram avaliados 54 pacientes com idade superior a 15 anos, com diagnóstico de traumatismo ocular fechado, assistidos no Pronto Socorro de Oftalmologia do Hospital São Paulo, no período de dezembro de 2000 a janeiro de 2002. O hifema foi classificado em cinco subgrupos: microscópico; grau I; grau II; grau III e grau IV (hifema total). Os pacientes foram divididos em dois grupos, de acordo com o comprometimento ou não do segmento posterior, para comparação dos dados pelo teste de Mann-Whitney e o teste exato de Fisher. A acuidade visual final foi avaliada por meio de regressão linear múltipla. Resultados: Noventa e um por cento dos pacientes eram do sexo masculino, com idade média de 32 anos. Na admissão, 37\% dos pacientes apresentaram PIO superior a $24 \mathrm{mmHg}$. O ressangramento ocorreu em $8 \%$ deles. Durante a evolução, seis pacientes necessitaram de intervenção cirúrgica. No grupo I (sem lesões de segmento posterior) houve melhora estatisticamente significante da AV ( $p<0,001)$, o que não foi observado no grupo II ( $p=0,4772)$. Conclusão: A classificação do hifema permite avaliação da gravidade da lesão, prognóstico e conduta. A baixa de visão persistente correlacionouse principalmente ao comprometimento do seguimento posterior e à acuidade visual na admissão. O sucesso do tratamento depende da identificação dos fatores de risco, medicação apropriada e indicação cirúrgica precisa.

Descritores: Traumatismos oculares; Hifema; Acuidade visual; Estudos prospectivos; Câmara anterior/lesões; Pressão intra-ocular/complicações; Seguimentos

Trabalho realizado no Departamento de Oftalmologia da Universidade Federal de São Paulo - UNIFESP.

Residente do Departamento de Oftalmologia da Universidade Federal de São Paulo - UNIFESP.

${ }^{2}$ Doutora, Setor de Trauma e Urgências do Departamento de Oftalmologia da Universidade Federal de São Paulo - UNIFESP.

${ }^{3}$ Mestre, Chefe do Setor de Trauma e Urgências do Departamento de Oftalmologia Universidade Federal de São Paulo - UNIFESP.

Endereço para correspondência: Av. Altino Arantes, 1132/132, São Paulo (SP) CEP: 04042-005

E-mail: karolinne@oftalmo.epm.br

Recebido para publicação em 10.12.2002

Versão revisada recebida em 20.05.2003

Aprovação em 30.05.2003

\section{INTRODUÇÃ̃O}

\begin{tabular}{l}
\hline INTRODUÇ̃̃o \\
\hline Hifema é uma apresentação freqüente em trauma ocular. Ocorre mais \\
comumente em jovens do sexo masculino ${ }^{(1)}$. Quando tratado de forma ade- \\
quada pode não deixar sequielas severas $^{(2)}$. Porém, complicações como \\
aumento da pressão intra-ocular e impregnação hemática da córnea podem \\
resultar em baixa acuidade visual final. A intensidade e mecanismo do \\
trauma estão diretamente relacionados ao prognóstico visual ${ }^{(3-4)}$. \\
O objetivo deste estudo foi avaliar a evolução de pacientes portadores \\
de hifema decorrente de trauma ocular contuso quanto à acuidade visual \\
(AV) inicial e final, aumento da pressão intra-ocular (Po), necessidade de \\
realização de procedimento cirúrgico, ocorrência de ressangramento e tem- \\
po de absorção do coágulo.
\end{tabular}




\section{MÉTODOS}

Foram avaliados 54 pacientes, retrospectivamente, com idade superior a 15 anos, com diagnóstico de traumatismo ocular fechado, assistidos no Pronto Socorro de Oftalmologia do Hospital São Paulo, no período de dezembro de 2000 a janeiro de 2002. Foram excluídos do estudo pacientes com hifema decorrente de trauma ocular aberto.

À admissão hospitalar, todos os pacientes foram submetidos a anamnese para coleta de dados (sexo, idade, tempo de trauma, objeto traumatizante), medida da acuidade visual (tabela de Snellen), exame biomicroscópico, tonometria de aplanação e fundoscopia. A gonioscopia com indentação foi realizada após quatro semanas do trauma ${ }^{(3)}$.

Ao exame inicial à lâmpada de fenda, o hifema foi classificado em cinco subgrupos: microscópico, quando observada presença de hemácias na câmara anterior sem a formação de nível; grau I, quando o nível atingiu menos de um terço da câmara anterior; grau II, com nível preenchendo um terço à metade da câmara anterior; grau III, com preenchimento de mais da metade da câmara anterior e grau IV, ou hifema total, com ocupação da totalidade da câmara anterior ${ }^{(2-4)}$.

Os pacientes foram orientados quanto ao repouso com decúbito a $30^{\circ}$ e aumento da ingesta hídrica. O uso de cicloplégicos, corticosteróides tópicos, beta-bloqueadores tópicos se PIO superior a $24 \mathrm{mmHg}$ foram padronizados. O retorno dos pacientes foi diário por pelo menos cinco dias.

As indicações para tratamento cirúrgico (lavagem da câmara anterior) foram: Po superior a $50 \mathrm{mmHg}$ por cinco dias, Po maior que $35 \mathrm{mmHg}$ por sete dias, hifema total com Po maior que $25 \mathrm{mmHg}$ por cinco dias e, nos casos de glaucoma prévio, impregnação de córnea e pacientes portadores de anemia falciforme com Po superior a $25 \mathrm{mmHg}$ por 24 horas $^{(3)}$.

Para fins de análise estatística, as acuidades visuais foram convertidas em logMAR e reconvertidas, ao final dos cálculos, para Snellen, quando conveniente. Os pacientes foram divididos em dois grupos; grupo I - ausência de comprometimento do segmento posterior e grupo II - com comprometimento do segmento posterior (edema de Berlim, hemorragia vítrea, descolamento de retina). Foram excluídos dessas análises os pacientes que se apresentaram com acuidades visuais piores ou iguais à percepção luminosa (PL-SPL). Para comparação entre os grupos em relação aos dados numéricos foi empregado o teste de MannWhitney; para os dados categóricos foi utilizado o teste exato de Fisher. A comparação de dados numéricos, dentro do mesmo grupo, foi realizada através do teste de Wilcoxon. Por meio de uma regressão linear múltipla realizou-se uma análise multivariada visando-se a avaliar as influências da acuidade visual inicial e grupo em relação à acuidade visual final. Para todas as análises foi adotado como nível de significância 0,05 .

\section{RESULTADOS}

Entre os 54 pacientes avaliados, 49 (91\%) eram do sexo masculino e $5(9 \%)$ do feminino. A idade apresentou variação de 15 a 72 anos, com média de 30 anos.
O tempo entre o trauma ocular e o atendimento inicial foi menor que 5 horas em 27 pacientes (50\%); em 8 casos (14\%) este tempo foi de 8 a 24 horas, em 13 (25\%) foi entre 2 e 5 dias, porém 6 pacientes $(11 \%)$ procuraram o PS somente após 10 dias do trauma.

$\mathrm{O}$ agente traumatizante mais frequiente foi espancamento, que inclui socos, garrafada, pedrada (20\%), seguido por bola (16\%), tampa de garrafa $(9 \%)$ e brinquedos como pistolas de plástico, bala de chumbo, estilingue e guidão de bicicleta $(9 \%)$.

$\mathrm{O}$ hifema traumático ocorreu mais freqüentemente em locais de lazer em $33 \%$ dos casos, seguidos por locais de trabalho (19\%), esporte (19\%), doméstico (19\%) e violência (assaltos, seqüestros-relâmpagos, brigas de rua) em $10 \%$.

Apenas 20 pacientes (37\%), na admissão, e 11 pacientes (20\%), durante a evolução, apresentaram Po superior a $24 \mathrm{mmHg}$, tendo sido prescrita medicação tópica hipotensora (betabloqueador).

Quatro pacientes apresentaram AV inicial SPL e sete apresentaram AV inicial PL na admissão. Os pacientes com AV inicial de SPL foram diagnosticados como portadores de avulsão do nervo óptico, neurite óptica isquêmica e traumática, enquanto os pacientes com AV inicial PL apresentavam lesões de segmento posterior (hemorragia vítrea e descolamento de retina).

O grupo I foi composto por 31 pacientes, os quais apresentaram lesões na córnea (edema, desepitelização, abrasão e ceratite puntata), hemorragia subconjuntival e facodonese. Foram encontradas lesões de segmento posterior (edema de Berlim, hemorragia vítrea e descolamento de retina) em 12 pacientes, os quais foram alocados no grupo II. A mediana da acuidade visual inicial dos pacientes do grupo I foi 20/63 e do grupo II foi $20 / 630$. Idade e sexo não diferiram entre os grupos (Tabela 1).

No diagrama de disperssão (Gráfico 1) que compara as AV inicial e final (logMAR), os pontos situados abaixo da linha diagonal representam melhora da $\mathrm{AV}$, os acima da linha representam piora e os sobre a linha nem melhora e nem piora. No grupo I houve melhora na grande maioria dos pacientes, di-

\begin{tabular}{|c|c|c|c|}
\hline & $\begin{array}{c}\text { Grupo I } \\
n=31\end{array}$ & $\begin{array}{c}\text { Grupo II } \\
n=12\end{array}$ & $p$ \\
\hline Idade (anos) & $32,10 \pm 13,91$ & $32,25 \pm 10,72$ & 0,6069 \\
\hline $\begin{array}{l}\text { Sexo } \\
\text { (masculino:feminino) }\end{array}$ & $28: 3$ & $10: 2$ & $0,6077^{\dagger}$ \\
\hline $\begin{array}{l}\text { AV inicial } \\
\text { (logMAR:Snellen) }\end{array}$ & $0,5: 20 / 63$ & $1,5: 20 / 630$ & 0,0191 \\
\hline $\begin{array}{l}\text { AV final } \\
\text { (logMAR:Snellen) }\end{array}$ & $0,1: 20 / 25$ & $1,0: 20 / 200$ & $<0,0001^{\circ}$ \\
\hline $\begin{array}{l}\text { Ganho de linhas } \\
\text { de visão }\end{array}$ & 4 & 0 & 0,0059 \\
\hline \multicolumn{4}{|c|}{$\begin{array}{l}\mathrm{AV}=\text { acuidade visual. Os valores relativos à } \mathrm{AV} \text { e ganho de linhas de } \\
\text { visão representam a mediana. Os valores relativos à idade representam a } \\
\text { média } \pm \text { desvio-padrão. } \\
\text { Teste de Mann-Whitney; }{ }^{\dagger} \text { Teste exato de Fisher }\end{array}$} \\
\hline
\end{tabular}




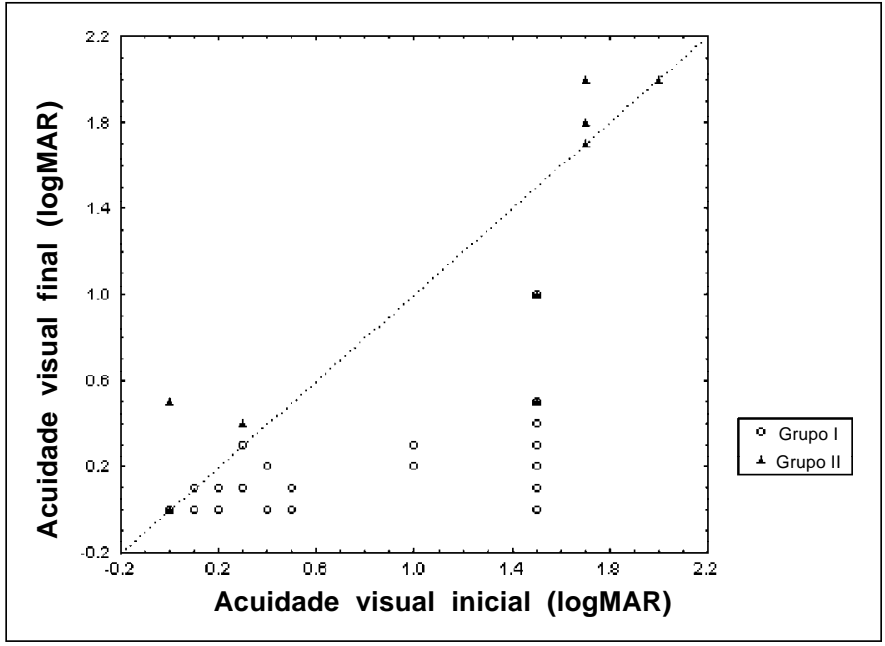

Gráfico 1 - Diagrama de dispersão representando as acuidades visuais iniciais e finais separadas por grupos

ferentemente do grupo II. Após o tratamento, em relação ao ganho de linhas de Snellen, houve melhora de quatro linhas (mediana) no grupo I, sendo que o grupo II não apresentou melhora.

No grupo I houve uma melhora estatisticamente significante da $\mathrm{AV}(\mathrm{p}<0,001)$, enquanto o grupo II não apresentou melhora significante $(\mathrm{p}=0,4772)$. A acuidade visual inicial $(\mathrm{p}<0,001)$ e o grupo $(\mathrm{p}<0,001)$ a que pertence o indivíduo foram preditores estatisticamente significantes e importantes (r2-72\%) da acuidade visual final.

Quanto ao subgrupo de hifema observado, $18 \%$ apresentavam hifema microscópico, $54 \%$ apresentavam hifema grau I, $12 \%$ grau II, $7 \%$ grau III e $9 \%$ foram classificados como hifema total.

O ressangramento ocorreu em $8 \%$ dos casos e esteve associado ao hifema inicial grau II. O tempo médio de clareamento foi de 9 dias, e também esteve associado à extensão do sangramento inicial. Dez pacientes (19\%) apresentaram impregnação da córnea como complicação.

Os pacientes foram seguidos até a resolução do quadro, em média durante 44 dias. Entretanto, os casos que apresentaram complicações foram acompanhados por até nove meses.

Seis pacientes necessitaram intervenção cirúrgica durante a evolução; três foram submetidos a paracentese e lavagem de câmara anterior (LCA) após o sexto dia de evolução e três pacientes foram submetidos à trabeculectomia (TREC) devido ao aumento crônico e refratário da Po por período superior a seis meses. Dos pacientes submetidos a TREC, dois já tinham sido submetidos a LCA.

\section{DISCUSSÃO}

O hifema traumático resulta de uma contusão ocular que causa um rápido aumento da pressão intra-ocular, estiramento do limbo, expansão da esclera equatorial, deslocamento pos-

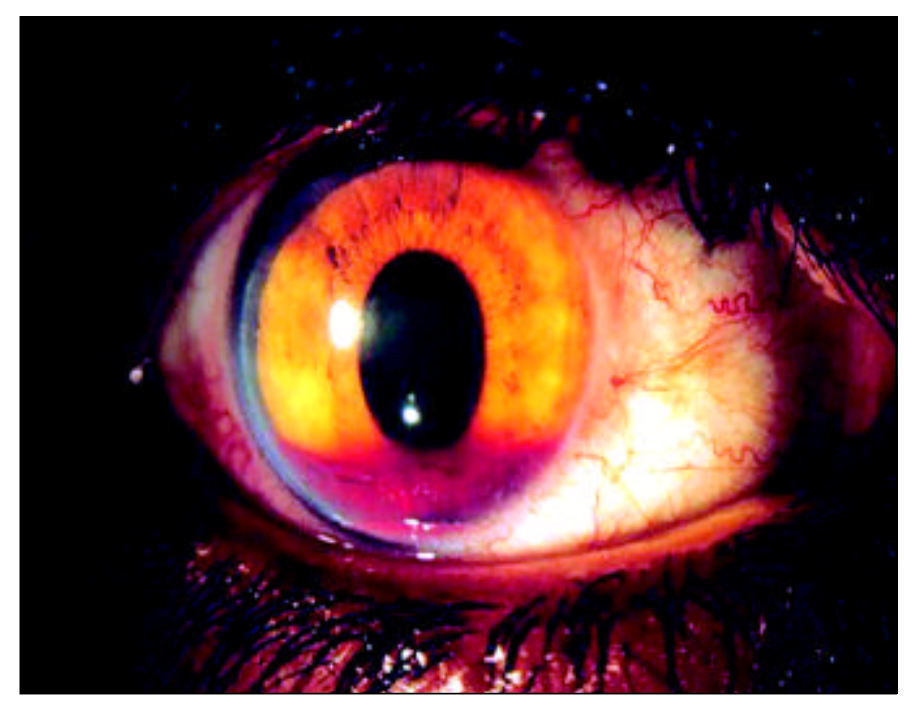

Figura 1. Paciente com hifema traumático grau I na admissão que evoluiu com acuidade visual final de 20/20

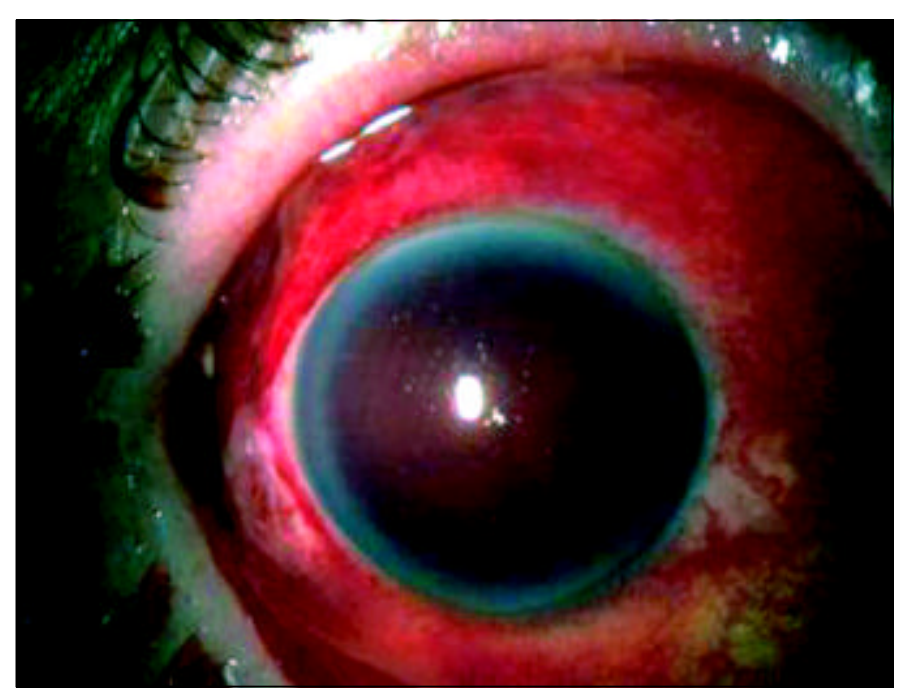

Figura 2. Paciente com hifema total (grau IV) que evoluiu com pressão intra-ocular superior a $25 \mathrm{mmHg}$ por 5 dias e foi submetido à paracentese e lavagem da câmara anterior

terior do cristalino e ruptura dos tecidos angulares ${ }^{(2-3)}$. O sangramento geralmente ocorre devido à lesão do círculo arterial maior da íris e de seus ramos, ramos do corpo ciliar ou arteríolas recorrentes coroidais. A hemorragia é tamponada pelo aumento da pressão intra-ocular, vasoespasmo e formação de coágulo de fibrina e plaquetas. A integridade máxima do coágulo é alcançada dentro de quatro a sete dias, sendo os produtos de degradação da fibrina drenados através do trabeculado ${ }^{(5)}$.

A classificação do hifema permite a avaliação da severidade da lesão, prognóstico e conduta. $\mathrm{O}$ volume de sangue na câmara anterior está relacionado à incidência de complicações $^{(3)}$. A acuidade visual final foi melhor que $0,7 \mathrm{em} 50 \%$ dos casos visto que o hifema traumático foi classificado como 
grau I em 54\% dos pacientes e como microscópico em $18 \%$. Dos pacientes que tiveram AV final melhor que 6/60, 147 (75\%) apresentavam hifema grau I e $9 \%$ dos pacientes apresentavam hifema microscópico ${ }^{(6)}$.

O tratamento do hifema ainda permanece controverso ${ }^{(4-5)}$. Preconiza-se repouso, decúbito a 30 graus e aumento da ingesta hídrica ${ }^{(3-4)}$. O uso de cicloplégicos pode ser benéfico previnindo a formação de sinéquias e permitindo maior conforto ao paciente. Os corticosteróides tópicos podem reduzir a irite traumática e em casos de aumento abrupto da pressão intraocular os hipotensores tópicos estão indicados. Os mióticos são contra-indicados, pois podem intensificar o processo inflamatório( ${ }^{(5)}$.

A incidência de ressangramento no estudo foi de $8 \%$, sendo a mesma estimada em $3,5 \%$ a $38 \%{ }^{(3-4)}$. O ressangramento ocorre mais freqüentemente entre o $2^{\circ}$ e $5^{\circ}$ dias pós-trauma conseqüente à lise e retração do coágulo ${ }^{(3)}$. Acuidade visual baixa e pressão intra-ocular elevada na admissão foram relacionadas à maior chance de ressangramento ${ }^{(6-7)}$. Estudos correlacionam o tamanho do hifema inicial com o risco de ressangramento $^{(8-9)}$, porém, o mesmo não foi confirmado por outros autores ${ }^{(6)}$. No presente trabalho, os pacientes que evoluíram com ressangramento apresentaram hifema inicial grau II ou maior.

Os anti-fibrinolíticos, como o ácido aminocapróico e o ácido tranexâmico, têm se mostrado eficazes na prevenção do ressangramento. Contudo, em populações com baixo risco de ressangramento, alguns autores não preconizam o uso rotineiro dos anti-fibrinolíticos devido ao seu alto custo e efeitos adversos ${ }^{(10)}$. No presente estudo, não adotamos a administração dos mesmos. Os agentes fibrinolíticos são indicados após um mínimo de 72h do trauma para os coágulos grandes e casos com risco de impregnação da córnea ${ }^{(11-12)}$. O uso de corticosteróides sistêmicos ainda não está bem estabelecido ${ }^{(13)}$. A hospitalização não parece diminuir a incidência de ressangramento ${ }^{(9)}$. A baixa acuidade visual final nos casos de hifema traumático resultou de comorbidades não afetadas pelo tratamento domiciliar.

Nos pacientes com glaucoma secundário e impregnação da córnea decorrentes de hifema traumático, a trabeculectomia parece ser um procedimento seguro nos casos em que a terapia medicamentosa falha no controle da pressão intra-ocular ${ }^{(14-15)}$. Os principais mecanismos de desenvolvimento do glaucoma secundário incluem: recessão angular, sinéquias periféricas anteriores, sinéquias posteriores com formação de íris "bombé", e glaucoma de células fantasmas. Três pacientes (6\%) evoluíram com aumento crônico e refratário da Po, correlacionandose à incidência estimada ${ }^{(5)}$, sendo submetidos à TREC sem intercorrências.

O prognóstico visual está relacionado à presença de lesões de segmento posterior ${ }^{(3,16)}$ e à acuidade visual na admissão, o que foi demonstrado por este estudo como estatisticamente significante $(\mathrm{p}<0,01)$. O sucesso do tratamento do hifema traumático, quando não há outras lesões mais graves associadas, depende da identificação dos fatores de risco, medicação apropriada e indicação cirúrgica precisa.

\section{CONCLUSÃO}

A baixa acuidade visual inicial e final ocorreu mais comumente devido a lesões do segmento posterior, catarata traumática e lesões traumáticas do nervo óptico do que do próprio hifema. A elevação da pressão intra-ocular decorrente do hifema traumático foi controlada por medicação tópica hipotensora na maioria dos casos, porém, três pacientes $(6 \%)$ foram submetidos à trabeculectomia decorrente do aumento crônico e refratário da Po. A incidência de ressangramento de $8 \%$ correlacionou-se à incidência estimada na literatura, mesmo sem o uso de medicações como o ácido aminocapróico e o ácido tranexâmico. A grande maioria dos pacientes evoluiu com resolução espontânea do hifema, entretanto, três pacientes $(6 \%)$ foram submetidos à paracentese e lavagem da câmara anterior sem intercorrências.

\section{ABSTRACT}

Purpose: To evaluate the evolution of the patients with hyphema resulting from blunt ocular trauma associated with initial and final visual acuity, elevated intraocular pressure, rebleeding, clot absorption and surgical intervention. Methods: Fifty-four patients aged more than fifteen years, with blunt ocular trauma attended on the Ophthalmology Emergency Room of São Paulo Hospital were studied, between December 2000 to January 2002. The hyphema was classified in to five groups: microscopic; grade I; grade II; grade III; and grade IV (total hyphema). The patients were divided into two groups according to the involvement or not of the posterior segment. The data were compared by MannWhitney and the exact Fisher test and the final visual acuity was evaluated by multiple linear regression. Results: Ninety-one percent of the patients were men with an average age of thirty two years. At admission, 37\% of patients showed intraocular pressure more than $24 \mathrm{mmHg}$. During the evolution, six patients required surgical intervention. Rebleeding occurred in $8 \%$ of the cases. In Group 1 (no lesions in the posterior segment) a better finally V.A. was statistically significant $(\mathrm{p}<0.001)$, a fact not observed in Group 2 ( $\mathrm{p}<0.4772$ ). Conclusion: Hyphema classification allows to evaluate the severity of damages, prognosis and management. The treatment still remains controversial. The final low visual acuity occurs more commonly associated with posterior segment injuries, and V.A. on admission. The successful treatment depends on identification of risk factors, appropriate medications and precise surgery indication.

Keywords: Eye injuries; Hyphema; Visual acuity; Prospective studies; Anterior chamber/injuries; Intraocular pressure/ complications; Follow-up studies

\section{REFERÊNCIAS}

1. Tongu MT, Bison SH, Souza LB, Scarpi MJ. Aspectos epidemiológicos do traumatismo ocular fechado. Arq Bras Oftalmol 2001;64:57-61.

2. Berríos RR, Dreyer EB. Traumatic hyphema. Int Ophthalmol Clin 1995;35:93-103. 
3. Shingleton BJ, Hersh PS. Traumatic hyphema. In: Shingleton BJ, Hersh PS, Kenyon KR. Eye trauma. St Louis: Mosby; 1998. p.104-14.

4. Walton W, Hagen SV, Grigorian R, Zarbin M. Management of traumatic hyphema. Surv Ophthalmol 2002;47:297-334.

5. Endo EG, Mead MD. The management of traumatic hyphema. Int Ophthalmol Clin 1994;34:1-7.

6. Rahmani B, Jahadi HR, Rajaeefard A. An analysis of risk for secondary hemorrhage in traumatic hyphema. Ophthalmology 1999;106:380-5.

7. Romano PE. Outpatient management of small traumatic hyphema. Eye 1994;8(Pt3):372.

8. Fong LP. Secondary hemorrhage in traumatic hyphema - Predictive factors for selective prophylaxis. Ophthalmology 1994;101:1583-8.

9. Shiuey Y, Lucarelli MJ. Traumatic hyphema - outcomes of outpatient management [commented on Ophthalmology 1998;105:2165]. Ophthalmology 1998;105:851-5.

10. Crouch ER, Williams PB, Gray MK, Chames M. Topical aminocaproic acid in treatment of traumatic hyphema [commented on Arch Ophtalmol 1997;115: 1189-90]. Arch Ophthalmol 1997;115:1106-12.
11. Laatikainen L, Mattila J. The use of tissue plasminogen activator in posttraumatic total hyphaema. Graefes Arch Clin Exp Ophthalmol 1996;234:67-8.

12. Starck T, Hopp L, Held KS, Marouf LM, Yee RW. Low-dose intraocular tissue plasminogen activator treatment for traumatic total hyphema, postcataract, and penetrating keratoplasty fibrinous membranes. J Cataract Refract Surg 1995;21:219-24.

13. Romano PE. Systemic prednisolone prevents rebleeding in traumatic hyphema. Ophthalmology 1999;106:375-9.

14. Graul TA, Ruttum MS, Lloyd MA, Radius RL, Hyndiuk RA. Trabeculectomy for traumatic hyphema with increased intraocular pressure. Am J Ophthalmol 1994;117:155-9.

15. Verma N. Trabeculectomy and manual clot evacuation in traumatic hyphema with corneal blood staining. Aust N Z J Ophthalmol 1996;24:33-8.

16. Rodrigues PD, Recalde PF, Morais NSB, Sousa LB, Freitas D. Prognóstico visual em pacientes com hifema traumático. Estudo prospectivo de 43 casos [resumo] Arq Bras Oftalmol 1994:57(4). Apresentado no XI Congresso Brasileiro de Prevenção da Cegueira e Reabilitação visual.

\section{Congresso da Sociedade Norte-Nordeste de Oftalmologia}

\section{0 de Junho a 3 de Julho de 2004 \\ Rio Połi Hołel - Teresina - PI}

\section{Informações: Agenda PromoçõesLtda. Fone/Fax: (86) 223-9444 E-mail: oftalmologia.nortenordeste.2004@bol.com.br Home-page: www.agendapromocoes.com.br}

